This item was submitted to Loughborough's Institutional Repository (https://dspace.lboro.ac.uk/) by the author and is made available under the following Creative Commons Licence conditions.

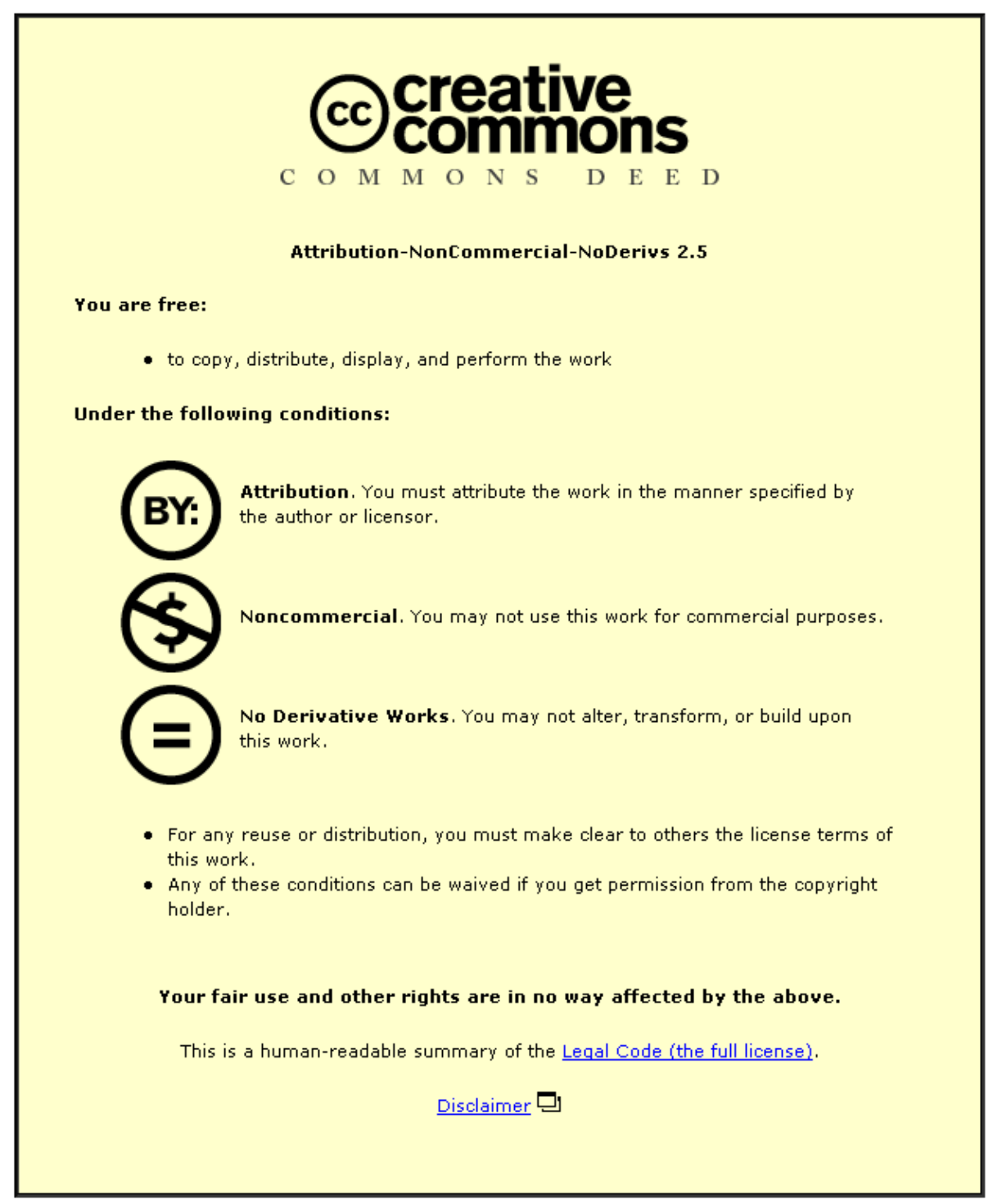

For the full text of this licence, please go to: http://creativecommons.org/licenses/by-nc-nd/2.5/ 


\title{
Characterising a solid state qubit via environmental noise
}

\author{
J.F.Ralph ${ }^{\mathrm{a}}$ T.D.Clark ${ }^{\mathrm{b}}$ M.J.Everitt ${ }^{\mathrm{b}}$ H.Prance ${ }^{\mathrm{b}}$ P.Stiffell $^{\mathrm{b}}$ \\ R.J.Prance ${ }^{\mathrm{b}}$ \\ ${ }^{a}$ Department of Electrical Engineering and Electronics, The University of \\ Liverpool, Brownlow Hill, Liverpool, L69 3GJ, United Kingdom. \\ ${ }^{\mathrm{b}}$ School of Engineering, The University of Sussex, Falmer, Brighton, BN1 9QT, \\ United Kingdom.
}

\begin{abstract}
We propose a method for characterising the energy level structure of a solid-state qubit by monitoring the noise level in its environment. We consider a model persistentcurrent qubit in a lossy resevoir and demonstrate that the noise in a classical bias field is a sensitive function of the applied field.
\end{abstract}

Key words: Quantum trajectory, Persistent current qubit PACS: 03.65.-w, 74.50.+r, 85.25.Dq

In this paper, we propose a novel technique to characterise the energy level structure of a solid state qubit and its spontaneous emission rate into a lossy reservoir. The technique relies on the backreaction of a solid state qubit on its environment and the incoherent transfer of energy from a high frequency mode to a low frequency mode due to the stochastic transitions of the qubit between energy eigenstates.

We consider a coupled system consisting of a model qubit and several classical degrees of freedom. The qubit is coupled to two main electromagnetic modes: a low frequency mode (classical bias field) that is used to control the operation of the qubit, and a higher frequency mode that is used to pump the qubit from the ground state to an excited state. In addition, the qubit is assumed to be coupled to a lossy reservoir, which represents the cavity that contains the qubit and control fields. The reservoir provides a mechanism to allow the qubit to dissipate energy and to induce spontaneous decays from an excited state into the ground state. The loss mechanism is modelled using a quantum trajectory approach $[1,2]$, corresponding to an unravelling of the Markovian Master 
equation for the qubit reduced density operator. In this paper, we choose the quantum jumps approach [1,2], which is suitable for modelling spontanteous emission processes and is computationally efficient [3]. Physically, this unravelling corresponds to the (irreversible) absorption of any spontaneously emitted photon on a time scale that is significantly faster than any of the time scales present in the quantum system. However, other unravellings of the Master equation can also be used to generate discrete stochastic jump-like behaviour and each process generates the usual reduced density evolution when averaged over an ensemble $[1,2,3,4]$.

Although these unravellings reproduce the Master equation evolution when averaged, they also contain other behaviour at the individual system level, such as chaotic-like behaviour that gives an indication of a classical limit that is not present in the reduced density operator [5]. Unfortunately, the general characteristics of evolution (jumps, chaos, etc.) are not specific to the unravelling and a large class of unravellings can have similar properties. As a result, the individual trajectories are normally considered to be subjective [2] by virtue of the fact that the predictive power of a simulation is contained in the average evolution, but care is required as to where the average is taken. In this paper, we consider the time-averaged evolution of a classical oscillator that is coupled reactively to a qubit that is described by a quantum jump model, as described in [3]. We demonstrate that the time-averaged behaviour of the classical oscillator can be used to characterise the energy level structure of the qubit and the rate of spontaneous decay into the reservoir. The behaviour is dependent on the presence of stochastic jump-like behaviour in the qubit, but it is not specific to the unravelling used.

The model qubit studied in this paper is a superconducting persistent current qubit proposed by Orlando et al. [6]. It is of particular interest because the states that would be used in an operational qubit device correspond to macroscopic current states, of the type that have been used in a series of experiments to demonstrate quantum properties of superconducting circuits: avoided-crossings in the energy level structure [7] and coherent oscillations of macroscopically distinct states [8]. Because of the macroscopic nature of the current states, it is also a system where the backreaction of the quantum device on the classical environment can be significant [9]. Although a persistent current qubit is studied in this paper, the same type of techniques could be adapted for other solid state qubits where the currents and/or voltages are comparable with those that are present in the classical control fields. The two-state Hamiltonian used in this paper is quite general and capacitive coupling between charge quibts could replace the inductive coupling used here. Small capacitance charge qubits can generate significant voltages from a small number of charges. A single electron on a charge qubit with a capacitance of $C \simeq 10^{-15} \mathrm{~F}$ would produce a voltage of around $V \simeq 0.2 \mathrm{mV}$, which is large enough to produce a significant backreaction on a classical field. 
The superconducting circuit described in reference [6] has been proposed as a candidate for the implementation of quantum processing in solid state devices. It is one of the most developed forms of persistent current qubit and similar devices having been used in recent experiments providing evidence for macroscopic coherent oscillations [8]. In this paper, we use the description of the system, together with the appropriate parameter values, given in reference [6] (see Figure 1). In this description, the qubit can be simplified to a two-state model corresponding to two current states which differ by approximately 600 $\mathrm{nA}$. The Hamiltonian for this system is given by,

$$
\hat{H}_{q u}\left(\Phi_{x 1}, \Phi_{x 2}\right)=\left(\begin{array}{cc}
F\left(\Phi_{x 1}, \Phi_{x 2}\right) & -B\left(\Phi_{x 1}, \Phi_{x 2}\right) \\
-B\left(\Phi_{x 1}, \Phi_{x 2}\right) & -F\left(\Phi_{x 1}, \Phi_{x 2}\right)
\end{array}\right)
$$

where

$$
\begin{aligned}
& F\left(\Phi_{x 1}, \Phi_{x 2}\right)=r_{1}\left(\frac{\Phi_{x 1}}{\Phi_{0}}\right)+r_{2}\left(\frac{\Phi_{x 2}}{\Phi_{0}}\right) \\
& B\left(\Phi_{x 1}, \Phi_{x 2}\right)=\frac{t_{1}+s_{1}\left(\frac{\Phi_{x 1}}{\Phi_{0}}\right)}{1-\eta \sqrt{\frac{E_{J}}{E_{C}}}\left(\frac{\Phi_{x 2}}{\Phi_{0}}\right)}
\end{aligned}
$$

and where the two principal control fields, the magnetic flux biases $\Phi_{x 1}$ and $\Phi_{x 2}$, are used in the operation of the qubit and may be time-dependant, and $\Phi_{0}=h / 2 e=2 \times 10^{-15} \mathrm{~Wb}$. The circuit specific constants are taken from [6]: $r_{1}=2 \pi E_{J} \sqrt{1-\frac{1}{4 \beta^{2}}}, r_{2}=r_{1} / 2, s_{1}=0, t_{1}=0.001 E_{J}, \eta=3.5, \beta=$ $0.8, E_{J} \equiv 200 \mathrm{GHz}, E_{C}=E_{J} / 80$. For the purposes of this paper, we will set $\Phi_{x 2}=0$ for convenience and introduce a relatively weak time-dependent field in $\Phi_{x 1}$ to induce transitions between the ground state and the excited state. We will typically use a time-dependent field of the form $\Phi_{h f 1}(t)=$ $0.00003 \Phi_{0} \cos (\omega t)$, with a frequency of $500 \mathrm{MHz}$. This applied field is the high frequency mode that we will use to drive the qubit, which - in turn - dissipates into the environment and transfers energy to the low frequency oscillator mode via an inductive backreaction. In theory, this drive could be coupled into the qubit via a circuit similar to the bias oscillator shown in Figure 1, but in practice it is more usual to drive the qubit devices at microwave frequencies using a coaxial waveguide [7]. For this reason, the high frequency mode is not modelled explicitly in this paper. The drive frequency is above the minimum splitting of the two states (which is $400 \mathrm{MHz}$ ) and excites transitions at a static flux bias of around $\Phi_{x 1}=0.00015 \Phi_{0}$, as shown in the insert in Figure 1. The insert in Figure 1 also shows a weak two photon transition, even for the very small amplitude drive used in this paper. Larger drive amplitudes can give rise to even higher order transitions due to the large nonlinearities present in Josephson junction circuits [10]. 


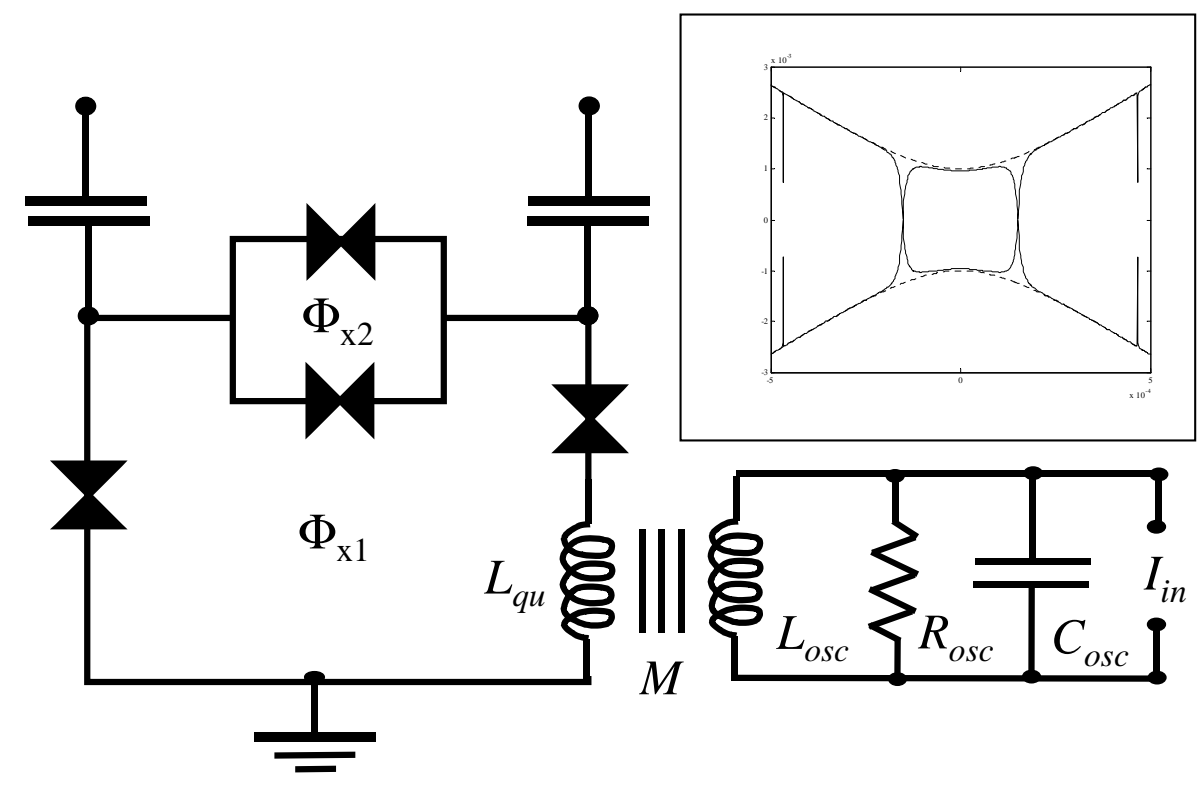

Fig. 1. Schematic diagram of persistent current qubit [6] inductively coupled to a (low frequency) classical oscillator. The insert graph shows the time-averaged (Floquet) energies as a function of the external bias field $\Phi_{x 1}$ for the parameters given in the text.

The qubit circuit is designed so that the inductance of the superconducting loop is negligible when compared with the effective inductance generated by the series Josephson junctions in the loop $\left(L_{J}=\Phi_{0} / 2 \pi I_{0}\right.$, where $I_{0}$ is the junction critical current). This means that the energy levels and the persistent current states of the circuit are dominated by the Josephson junctions rather than the geometrical inductance of the ring, $L_{q u} \simeq 10 \mathrm{pH}$ [6]. However, the inductance does play an important role when determining the coupling between the ring and the the external fields. In inductively coupled circuits, the magnetic fluxes are related to the currents flowing through the inductors by the inductances and the mutual inductances. For the qubit coupled to the low frequency oscillator, this gives

$$
\begin{aligned}
& \Phi_{q u}=L_{q u} I_{q u}+M I_{o s c} \\
& \Phi_{o s c}=L_{o s c} I_{o s c}+M I_{q u}
\end{aligned}
$$

where $M$ is the mutual inductance, $\Phi_{\text {osc }}$ is the magnetic flux in the oscillator and the oscillator is characterized by a capacitance $C_{o s c}$, and an inductance $L_{\text {osc }}$. In the absence of dissipation (represented by the parallel resistance in the oscillator circuit) the effective classical Hamiltonian for the combined system can be written in the form [11],

$$
H=\frac{Q_{o s c}^{2}}{2 C_{o s c}}+\frac{\Phi_{o s c}^{2}}{2 L_{o s c}}-\Phi_{o s c} I_{i n}+\left\langle\hat{H}_{q u}\left(\mu \Phi_{o s c}+\Phi_{h f 1}(t), \Phi_{x 2}\right)\right\rangle
$$


where $I_{\text {in }}$ is the external current applied to the oscillator and the coupling coefficients are given by $K^{2}=M^{2} / L_{q u} L_{o s c}$ and $\mu=M / L_{o s c}$. An additional effect of the inductive coupling is to shift the effective geometric inductance of the superconducting loop by a factor $\left(1-K^{2}\right), L_{q u} \rightarrow L_{q u}\left(1-K^{2}\right)[11]$.

In the presence of dissipation, the classical equation of motion for the oscillator becomes

$$
C_{o s c} \frac{d^{2} \Phi_{o s c}}{d t^{2}}+\frac{1}{R_{o s c}} \frac{d \Phi_{o s c}}{d t}+\frac{\Phi_{o s c}}{L_{o s c}}=I_{i n}+\frac{\mu\left\langle\hat{I}_{q u}\left(\mu \Phi_{o s c}+\Phi_{h f 1}(t), \Phi_{x 2}\right)\right\rangle}{\left(1-K^{2}\right)}
$$

where we have inserted the parallel resistance $R_{\text {osc }}$, and the qubit screening current in the SQUID ring is calculated at each point in time from the expectation value of the qubit screening current operator $\hat{I}_{q u}$ over the instantaneous wavefunction (i.e. a pure state) of the qubit using the bare (unrenormalised) inductance of the qubit, $L_{q u}$. The use of the expectation value in a classical equation of motion is a reasonable approximation in situations where the oscillator has a lower frequency than any of the frequencies present in the qubit and where any quantum fluctuations present in the oscillator are negligible compared to its classical evolution. It is consistent with the general approach to quantum trajectories and the classical limit, and retains the subjective nature of the trajectories because the exact details of the quantum evolution of the qubit cannot be reconstructed from the time-averaged classical behaviour of the oscillator. Here, we use a noise-driven oscillator with a resonant frequency of $\omega_{o s c}=1 / \sqrt{L_{o s c} C_{o s c}}=300 \mathrm{MHz}$, a capacitance of $C_{o s c}=1 \mathrm{nF}$, and a quality factor of $Q_{o s c}=\omega_{o s c} R_{o s c} C_{o s c}=188$. The oscillator frequency is chosen to be $300 \mathrm{MHz}$ so that there are no harmonic relationships between the oscillator and any of the frequencies within the region of interest. The input current that is used to drive the oscillator $I_{i n}=I_{d c}+I_{n o i s e}(t)$ consists of a dc current (which can be used to bias the qubit, $\Phi_{d c}=L_{o s c} I_{d c}$ ) and a timevarying component due to Johnson noise in the resistor at finite temperature, $T=4$ Kelvin. (The noise need not be thermal, but it is a useful generic model for experimental noise because electronic noise is often characterised in terms of an effective noise 'temperature'). The coupling between the qubit and the oscillator is very weak, $K=0.01$ giving $\mu=0.002$, to prevent any distortion of the apparent qubit resonance due to a static backreaction [9].

The quantum evolution of the qubit is determined by three competing effects: the high frequency drive field, the noise-driven fluctuations in the coupled oscillator, and the effect of spontaneous emission of the qubit into the lossy reservoir. The drive field will induce transitions (Rabi oscillations) in the qubit when the bias field is close to a quantum resonance. The noise present in the low frequency oscillator will introduce dephasing (destroying the absolute phase reference of any coherent oscillations) and a small amount of dissipation due to the presence of the resistive element in the oscillator circuit (the second 

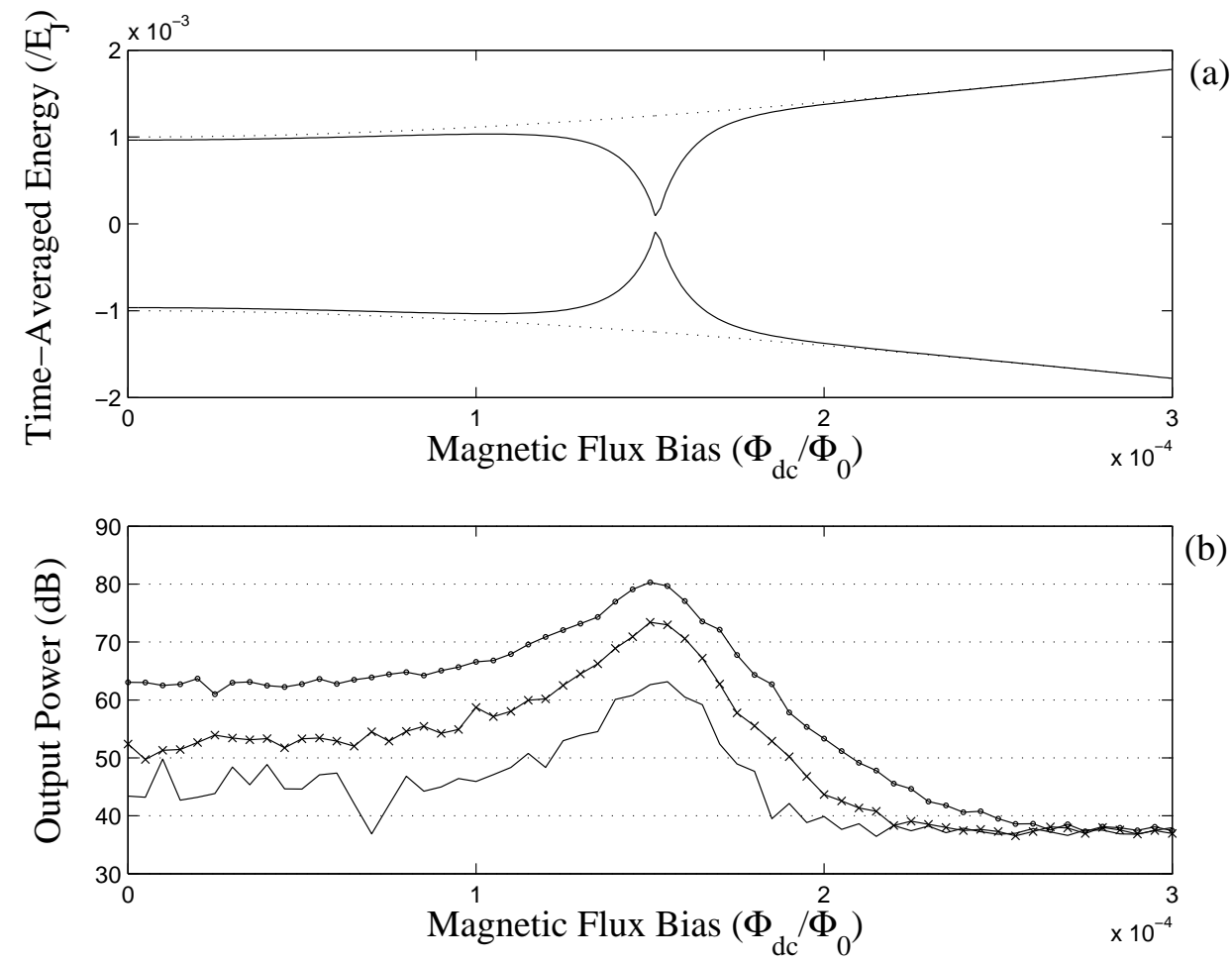

Fig. 2. (a) Close-up of the time-averaged (Floquet) energies of the single photon resonance $(500 \mathrm{MHz})$ - solid lines - with the time-independent energies given dotted lines. (b) The output power of the low frequency oscillator at $300 \mathrm{MHz}$, as a function of the static magnetic flux bias: $\gamma=0.005$ per cycle (solid line), $\gamma=0.05$ per cycle (crosses), $\gamma=0.5$ per cycle (circles). The other parameters are given in the text

effect is small by virute of the weak coupling). The spontaneous emission process will introduce quantum jumps that project into the instantaneous ground state of the qubit. During time intervals where no spontaneous decay is produced, the evolution contains terms due to coherent Schrödinger evolution and non-Unitary terms, as described in reference [3]. During a small - but finite time interval, $d t$, the probability of a spontaneous decay occuring is $\gamma\left\langle\hat{c}^{\dagger} \hat{c}\right\rangle d t$, where $\hat{c}^{\dagger}$ and $\hat{c}$ are the raising and lowering operators for the qubit states respectively. If a spontaneous decay occurs, an operator $\hat{\Omega}_{1}(d t)=\sqrt{\gamma d t} \hat{c}$ is applied to the qubit wavefunction. If no spontaneous decay occurs, an evolution operator

$$
\hat{\Omega}_{0}(d t)=1-\frac{i}{\hbar} \hat{H}_{q u} d t-\frac{\gamma}{2} \hat{c}^{\dagger} \hat{c} d t
$$

is applied. The decay rate $\gamma$ is fixed by the coupling of the qubit to the lossy reservoir.

The evolution of the system is determined by numerical integration of the equations of motion (classical and quantum jump), using random initial states and ignoring the initial transient motion. The output is chosen to be the voltage fluctuations in the classical oscillator, which can either be characterised 
by the time-averaged root mean square (RMS) power, or the power spectral density near a particular frequency. Figure 2 shows three examples of the peak power in the main oscillator resonance (near to $300 \mathrm{MHz}$ ) for different values of the spontaneous decay rate $\gamma=0.005,0.05,0.5$ per oscillator cycle. In each of the graphs there is an asymmetry either side of the resonance. This is due to the fact that the resonance is quite near to the minimum splitting point and the energy eigenstates contain a significant contribution from both current states near the minimum splitting (to the left of the graph) and are predominately one current state on the right of the resonance. Moving away from the minimum splitting point (increasing the frequency of the applied field) restores the symmetry of the peaks. (The apparent noise on the bottom graph is due to the fact that the averaging process is limited in time and the damping rate is sufficiently low that comparatively few quantum jumps are seen).

In each of the cases shown in Figure 2, there is a significant increase in output power near to the resonance, between 10 and $20 \mathrm{~dB}$ between the left and the peak and between 20 and $40 \mathrm{~dB}$ between the right and the peak. When the spontaneous decay rate is reduced the power gain is reduced. Interestingly, there is no gain in output power at the resonant frequency of the oscillator when there are no spontaneous emissions $(\gamma=0)$ and the output power is not a function of the applied static flux. This is because the discontinuous jumps in the qubit are coupled back to the oscillator, and act as an additional source of noise, thereby increasing the output power. Near to the resonance, when the qubit is more likely to be in the excited state, and therefore more likely to undergo a quantum jump, the noise introduced by the jumps is enhanced. Clearly, the output voltages near the peaks shown in Figure 2 are significantly above the thermal fluctuations in the oscillator ( 4 Kelvin $\simeq 40 \mathrm{~dB}$ in the units used here) and their detection would allow the magnetic flux bias of the resonance to be determined. The model does not include any direct coupling between the low frequency and the high frequency modes, which is likely to be present in an experimental system, but this coupling will be independant of the static flux bias, so it is only the changes in the output power as a function of the static flux that concern us here.

By detecting the position of the peak in the output voltage/power for different frequencies of the drive field, it should be possible to determine the energy level separations of the two states as a function of static magnetic flux. The accuracy of the flux position would be determined by the width of the resonance, and consequently the amplitude of the applied drive field. The larger the high frequency drive, the broader the resonance. For the example studied here the flux accuracy is of the order of $10^{-5} \Phi_{0}$. The accuracy of the frequency applied to the qubit is determined by the stability of the classical (mono-frequency) source, but at the frequencies used a typical value would be of the order of a few Hz. However, it should be noted that the conventional method for applying 

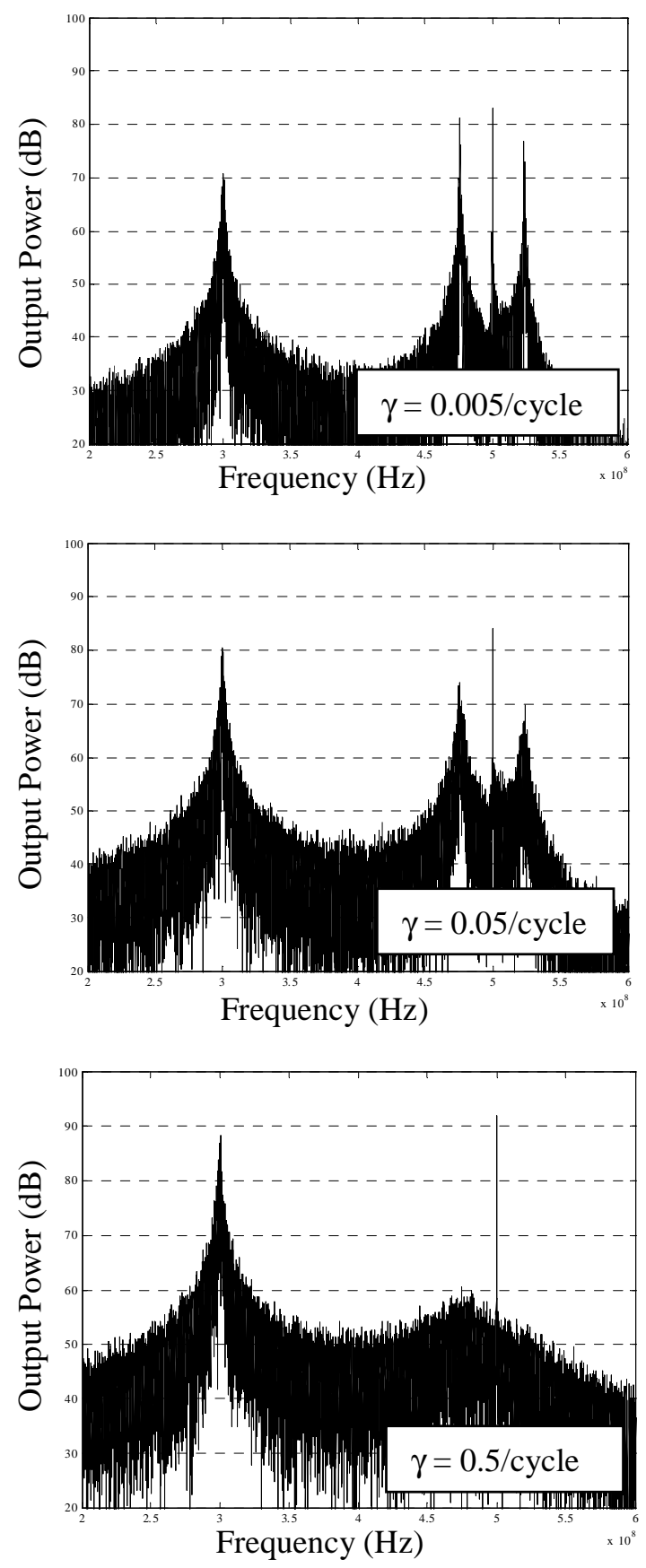

Fig. 3. Power spectral density for the low frequency oscillator at the resonance point $\left(\Phi_{d c}=0.00015 \Phi_{0}\right)$ for the three spontaneous decay rates shown in Figure 2 : $\gamma=0.005,0.05,0.5$ per cycle. The other parameters are given in the text

high frequency fields (coaxial waveguides [7]) is not best suited to this process because the coupling between the qubit device and the applied drive field may vary with frequency. This will not change the position of the output peak, but it will change the shape of the peak because the strength of the coupling will alter the width of the resonance. 
Figure 3 shows the power spectral density of the output from the oscillator for the three different decay rates shown in Figure 2. The main resonance can be seen in each graph at $300 \mathrm{MHz}$, as well as the fraction of the $500 \mathrm{MHz}$ drive that couples directly from the high frequency mode to the low frequency mode via the qubit. The other peaks that can be seen either side of the 500 $\mathrm{MHz}$ peak in the first two graphs are sidebands generated by mixing the Rabi oscillation frequency with the drive frequency. The Rabi oscillation frequency for the case considered here is much less than the resonant frequencies of the modes, $f_{\text {Rabi }} \simeq 25 \mathrm{MHz}$. In the first two graphs, the coupling to the lossy reservoir is sufficiently low for the Rabi oscillations to occur (more or less) undisturbed by the quantum jumps. The larger linewidth in the second graph indicating the larger spontaneous decay rate. In the third graph, there is no evidence of these Rabi sidebands because the average spontaneous emission frequency is much smaller than the Rabi frequency. Whilst the large signal gain shown in Figure 2, is an indication of the presence of a quantum transition, it does not indicate that the behaviour of the qubit is dominated by coherent Schrödinger evolution. The presence of Rabi sidebands is a good indication that the behaviour of the system is predominantly coherent and the linewidth of these sidebands provides an indication of the damping rate. The position of the sidebands relative to the drive frequency also gives a good estimate of the Rabi frequency.

In this paper, we have discussed the effect of resonant transitions in a model qubit on a low frequency oscillator. The qubit is coupled to two modes and a lossy reservoir: one high frequency mode used to drive the transitions between energy eigenstates of the qubit, and one low frequency oscillator that is used to control the bias field for the qubit. The coupling to the lossy reservoir induces spontaneous decay of the qubit from an excited state to its instantaneous ground state. Near a quantum mechanical resonance between the high frequency mode and the qubit, the qubit is more likely to be in an excited state and quantum jumps introduced by the spontaneous emission are more frequent. The discontinuous jumps in the qubit are coupled to the low frequency oscillator via the mutual inductance and give rise to significant increases in the output power detected in the oscillator when the qubit is biased near to a quantum resonace. We have investigated the effiect of this backreaction on the low frequency oscillator and suggested how this power gain could be used to characterise the energy level structure of the qubit and its coupling to the lossy reservoir.

The authors would like to thank the Engineering and Physical Science Research Council (EPSRC) Quantum Circuits Network for their generous support. 


\section{References}

[1] H.J.Carmichael, 'An Open System Approach to Quantum Optics' (Lecture Notes in Physics, Vol.18), Springer-Verlag, Berlin, 1993;

[2] H.M.Wiseman, Quant. Semiclass. Opt. 8, 205 (1996).

[3] H.M.Wiseman, G.E.Toombes, Phys. Rev. A 60, 2474 (1999).

[4] N.Gisin, I.C.Percival, J. Phys. A 26, 2233 (1993); N.Gisin, I.C.Percival, J. Phys. A 26, 2246 (1993); G.C.Hegerfeldt, Phys. Rev. A 47, 449 (1993).

[5] T.P.Spiller, J.F.Ralph, Phys. Lett. A 194, 235 (1994); T.A.Brun, I.C.Percival, R.Schack, J.Phys. A 292077 (1996).

[6] T.P.Orlando, J.E.Mooji, L.Tian, C.H. van der Wal, L.S.Levitov, S.Lloyd, J.J.Mazo, Phys. Rev. B 60, 15398 (1999).

[7] J.R.Friedman, V.Patel, W.Chen, S.K.Tolpygo, J.E.Lukens, Nature 406, 43 (2000); C.H. van der Wal, A.C.J. ter Haar, F.K.Wilhem, R.N.Schouten, C.J.P.M.Harmans, T.P.Orlando, S.Lloyd, J.E.Mooij, Science 290, 773 (2000).

[8] I.Chiorescu, Y.Nakamura, C.J.P.M.Harmans, J.E.Mooij, Science 299, 1869 (2003).

[9] J.F.Ralph, T.D.Clark, M.J.Everitt, P.Stiffell, Phys. Rev. B. 64, 180504 (2001)); J.F.Ralph, T.D.Clark, M.J.Everitt, P.Stiffell, R.J.Prance, H.Prance, Proceedings of SPIE conference 'Quantum Computing III', SPIE Vol. 4732 Eds. A.R.Pirich, E.W.Taylor, E.Donkor (2002).

[10] T.D.Clark, J.Diggins, J.F.Ralph, $\quad$ M.J.Everritt, R.J.Prance, H.Prance, R.Whiteman, Ann. Phys. 268, 1 (1998); M.J.Everitt, T.D.Clark, P.Stiffell, H.Prance, R.J.Prance, A.Vourdas, J.F.Ralph, Phys. Rev. $B$ 64, 184517 (2001).

[11] J.F.Ralph, T.P.Spiller, T.D.Clark, R.J.Prance, H.Prance, Int. J. Mod. Phys. B 8, 2637 (1994); J.Diggins, J.F.Ralph, T.D.Clark, T.P.Spiller, R.J.Prance, H.Prance, Phys. Rev. E 49, 1854 (1994); T.D.Clark, J.F.Ralph, R.J.Prance, H.Prance, J.Diggins, R.Whiteman, Phys. Rev. E 57, 4035 (1998). 